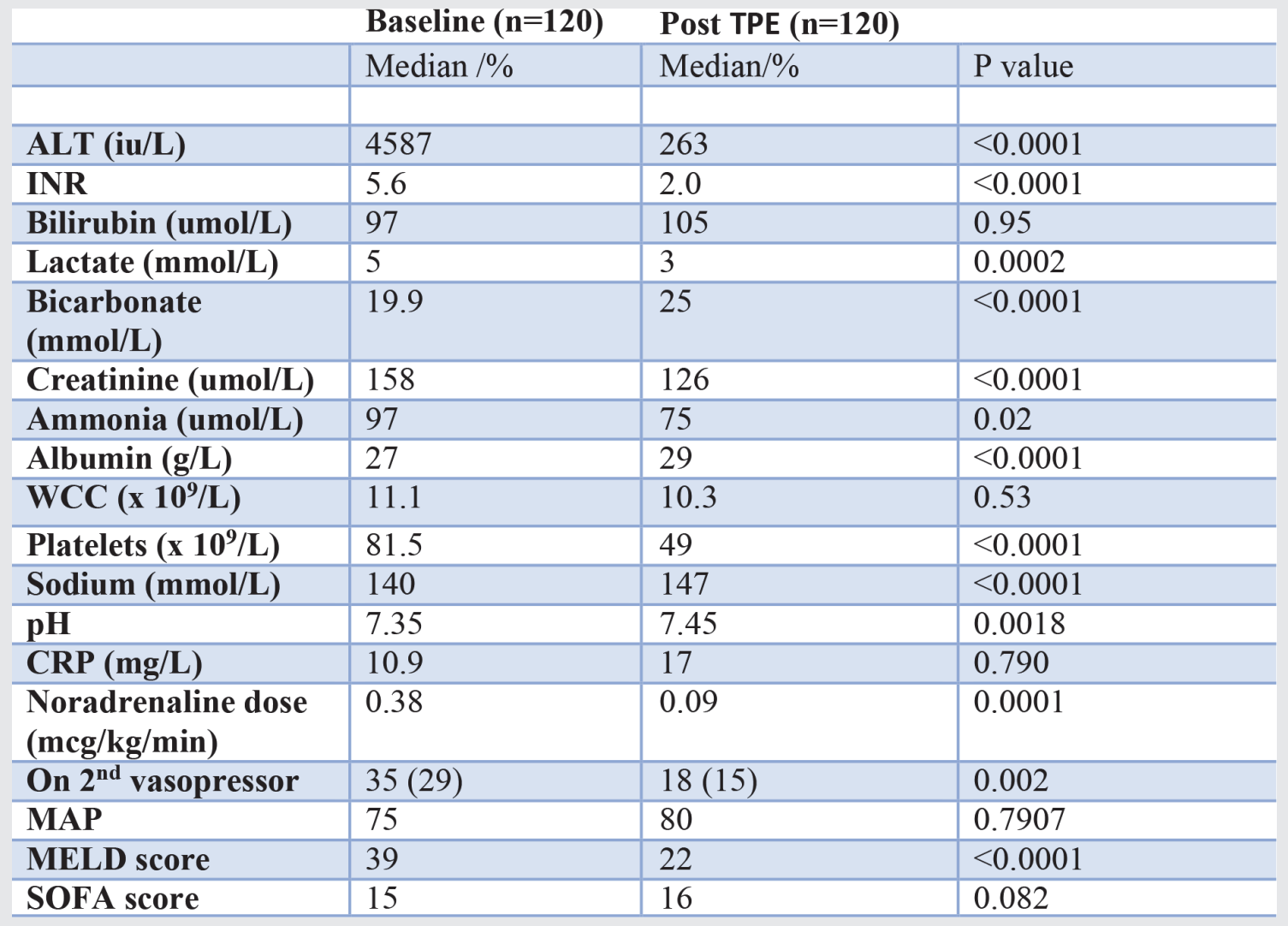

76.6\% of patients met Kings College Criteria (KCC) for poor prognosis. Median number of TPE sessions performed was 3 (IQR 2-3), with a median volume of 4 litres exchanged (IQR 3-5litres). Table 1 shows the changes in clinical variables pre and post TPE. Significant improvements $(p<0.05)$ were seen in the majority of clinical and biochemical variables. Notably there was a reduction in noradrenaline doses $(0.4 \mathrm{mcg} / \mathrm{kg} / \mathrm{min}$ to $0.09 \mathrm{mcg} / \mathrm{kg} / \mathrm{min}, \mathrm{p}=0.0001$ ) and in requirements for a second vasopressor agent $(29 \%$ to $15 \%, p=0.002)$. This benefit remained when subgroup analysis of patients fulfilling poor prognostic KCC was performed. Figure 1 shows in those meeting KCC overall survival was 50\%. Transplant free survival was $36 \%$; higher than seen in previous cohorts of patients fulfilling KCC and receiving standard medical treatment.

Conclusion LV TPE improves clinical variables of patients with ALF comparable with results seen in HV TPE. TPE remains a promising supportive therapy in creating a haemodynamic window of stability for transplant and as an alternative therapy for patients unsuitable for transplantation.

\section{P068 CASE FINDING FOR HEPATIC FIBROSIS IN AT-RISK POPULATIONS - ENHANCED LIVER FIBROSIS SCORE DETECTS MORE IN ALCOHOL THAN NONALCOHOLIC FATTY LIVER DISEASE}

${ }^{1}$ Alex Hinkson*, ${ }^{1}$ Jacob Feathers, ${ }^{1}$ Jason Punnamkuzhy, ${ }^{1}$ Firuza Dzhakhangirli, ${ }^{1,2}$ lan AC Rowe, ${ }^{1}$ Richard Parker. 'Leeds Teaching Hospitals NHS Trust, Leeds, UK; ${ }^{2}$ Leeds Institute for Data Analytics, University of Leeds, Leeds, UK

10.1136/gutjnl-2021-BASL.77
Background Nonalcoholic fatty liver disease (NAFLD) and Alcohol-related liver disease (ALD) are the most common causes of chronic liver disease in the UK. Early detection of fibrotic liver disease can allow intervention aiming to reduce progression. We report on results of case finding in at-risk groups in community settings to identify patients with advanced liver fibrosis.

Method Patients undergoing diabetic health checks at in primary care, or patients with high-risk alcohol intake in alcohol treatment services were tested for liver fibrosis using the enhanced liver fibrosis (ELF) test. Patients with ELF $<9.5$ were deemed at low risk of fibrosis and not examined further. Patients with ELF $>9.5$ were referred for liver stiffness measurement (LSM) by FibroscanTM and clinical review. $\mathrm{LSM}>15 \mathrm{kPa}$ was considered indicative of advanced disease.

Results A total of 1092 patients were included: 518 patients with diabetes and 574 patients with high-risk alcohol intake. In the diabetic cohort $54.6 \%$ had ELF $>9.5$, compared with $28.2 \%$ in the alcohol cohort. LSM was done in 246 patients with diabetes and 117 patients with high-risk alcohol intake. The median LSM result was $6.2 \mathrm{kPa}$ in the diabetic cohort and $8.9 \mathrm{kPa}$ in the alcohol cohort. In the diabetic cohort, $7.3 \%$ of LSM results were $>15 \mathrm{kPa}$, compared to $30.8 \%$ in the alcohol cohort. 438 patients had LFTs done within 3 months of ELF testing, 181 of these had ELF >9.5 and 96 (53\%) had a normal ALT.

Conclusion Case finding in persons with diabetes in primary care yielded relatively few positive cases. In contrast, over a quarter of cases with high-risk alcohol intake identified with blood tests had advanced disease. Importantly, ALT is normal in most patients with advanced disease confirming that 
standard liver blood tests are inadequate to detect clinically silent disease. We conclude that one-stage testing may be appropriate in populations with high prevalence of underlying disease, such as high-risk alcohol intake, whereas further discrimination is required in cohorts with a lower prevalence.

\section{P069 CREATING A SURROGATE ARTIFICIAL LIVER USING 3D PRINTING AND MECHANICAL CHARACTERISATION OF HEPATIC TISSUES FOR TUMOUR REMOVAL EDUCATION}

${ }^{1}$ Richard Arm*, ${ }^{1}$ Arash Shahidi, ${ }^{2}$ Chris Clarke. ${ }^{1}$ Nottingham Trent University, Nottingham, UK; ${ }^{2}$ Nottingham University Hospital, Nottingham, UK

\subsection{6/gutjnl-2021-BASL.78}

This research aims to identify suitable synthetic materials and manufacturing methodologies that will enable the production of accurate, patient-specific, anthropomorphic surrogate organs to allow surgeons to rehearse tumour removal using traditional or robotic techniques prior to the live surgery.

Shore hardness $(\mathrm{H} 00)$ and elastic modulus (MPa) of both porcine tissues and surrogate tissues were tested with the same equipment and methods. We used international test standards (ASTM D 2240-03 and BS 903-A26:1995/ISO 48:1994, BS/ISO 23529:2016, ASTMD412 AND BS/ISO $37: 2011)$. The hardness data was collected using a shore 00 calibrated durometer. Stress versus strain curves were plotted using the data collected from a Zwick/Roell tensile testing machine. Both pieces of equipment were used to characterise and engineer synthetic soft tissues to match the existing data and observations of porcine liver. CT and MRI DICOM data was used to produce multiplanar reconstructions and rigid $3 \mathrm{D}$ printed artefacts of liver anatomy (blood vessels, tumour, and parenchyma). The models were transformed into physiological counterparts using multi layered synthetic gels and fibres that mimic variable tissue properties like hardness and elastic modulus based on data of human liver given in literature and our porcine liver observations.

Previously published hardness of live human parenchyma was $15.06 \mathrm{H} 00( \pm 2.64)$, while we found porcine liver parenchyma to be $23 \mathrm{H} 00$ ( \pm 0.1 ). Hardness of the synthetic parenchyma of liver was $10 \mathrm{H} 00( \pm 2.3)$.

Hardness of porcine vascular tissue thickness did not meet requirements for agreed test standard, but suitable bovine aortic tissue was previously investigated and was found to be $41 \mathrm{H} 00$. The hardness of our surrogate vascular tissue was 40 H00. No data exists on the hardness of tumours for comparison.

Literature shows the elastic Young's modulus of healthy adult livers to be between $0.01-0.015 \mathrm{MPa}$. The porcine specimens had a modulus of $0.02-0.08 \mathrm{MPa}$ within $0.2-0.4$ strain range. These specimens failed at a force of $7.33 \mathrm{~N}$. The surrogate modulus was $0.006-0.02 \mathrm{MPa}$ within 1-1.7 strain range and failed at $0.67 \mathrm{~N}$.

Surrogate liver tissues had similar hardness and elastic modulus to human and porcine equivalents. We found porcine parenchyma to be more brittle, less extensible and slightly stiffer than both human and synthetic surrogate tissue. Production methodology of surrogate liver and internal vasculature was successful, but better resolution of the smaller vessels $(<2 \mathrm{~mm})$, refinement of the additive manufacturing process and methods of multi-layered lamination might improve models.

\section{P070 LIVER STIFFNESS MEASUREMENT PERFORMS POORLY IN HAEMOCHROMATOSIS}

${ }^{1}$ Alex Hinkson*, ${ }^{2}$ Guy Gitlin-Leigh, 'Joanna Moore. 'Leeds Teaching Hospitals NHS Trust, UK; ${ }^{2}$ Leeds School of Medicine, University of Leeds, Leeds, UK

\subsection{6/gutjnl-2021-BASL.79}

Background Haemochromatosis can lead to liver fibrosis and cirrhosis. Liver biopsy is the gold standard for assessing fibrosis but is associated with risks to the patient. Non-invasive techniques are increasingly used for staging of fibrosis. Liver Stiffness Measurement (LSM) is commonly used in other disease aetiologies but performance in haemochromatosis is not well characterised. Our aim was to determine whether LSM reliably detects advanced fibrosis or cirrhosis in patients with haemochromatosis.

Methods This study was a retrospective analysis of data from patients being treated for haemochromatosis in Secondary Care who underwent LSM by Fibroscan ${ }^{\mathrm{TM}}$ and had biopsy for characterisation and staging of disease. Patients without liver biopsy were excluded. Data regarding genotype, biochemical parameters, biopsy indication, histology and LSM were collected. Patients with cirrhosis on biopsy were assumed to remain cirrhotic. Patients with more than 18 months between biopsy and LSM were excluded. Median LSM values for different patient groups were calculated in Excel. A planned ROC analysis was abandoned due to insufficient patient numbers.

Results Data from 25 patients with liver biopsy were collected. All these patients had LSM. Median LSM for all patients in our cohort was $8.5 \mathrm{kPa}$. Only 6 patients had LSM within 18 months of biopsy, of whom 2 had bridging fibrosis and 5 had peri-portal or no fibrosis. Median LSM for these patients was $9.8 \mathrm{kPa}$ and mean was $11.1 \mathrm{kPa}$. Six patients had histological evidence of cirrhosis. Median LSM for this group was $9.1 \mathrm{kPa}$ and mean was $9.8 \mathrm{kPa}$.

Conclusion Our data suggest that LSM may not be a reliable tool for quantifying fibrosis in patients with haemochromatosis, as those with low levels of fibrosis often had LSM values higher than patients with known cirrhosis. These results may

\begin{tabular}{llll} 
Abstract P070 & Table 1 & & \\
\hline Patient number & LSM/Biopsy interval & Fibrosis Stage & Median LSM (kPa) \\
\hline 1 & 1 months & No bridging & 10 \\
2 & 12 years & Cirrosis & 22.5 \\
3 & 3 months & Bridging fibrosis & 9.6 \\
4 & 2 months & Bridging Fibrosis & 7.6 \\
5 & 10 years & Cirrhosis & 10.3 \\
6 & 6 weeks & No bridging & 12.4 \\
7 & 13 days & No bridging & 3.7 \\
8 & 4 years & Cirrhosis & 7.1 \\
9 & 3 months & No bridging & 2.8 \\
10 & 14 years & Cirrhosis & 6.4 \\
11 & 10 years & Cirrhosis & 9.1 \\
12 & 10 years & Cirrhosis & 9.1 \\
\hline
\end{tabular}

